\title{
A zinc transporter protects from ischemia-reperfusion injury
}

\author{
Friedrich C. Luft
}

Published online: 25 January 2012

(C) Springer-Verlag 2012

Intuitively, matters should promptly improve after blood supply is restored to a previously ischemic tissue; however, the reverse is the case. A series of events leads to inflammation and oxidative damage through the induction of oxidative stress, rather than restoration of normal function. White blood cells carried to the area by the returning blood release a host of inflammatory factors, including cytokines and free radicals, in response to tissue damage. The restored blood flow brings with it vital oxygen that also contributes to DNA, protein, and cellular membrane damage. Apoptosis is stimulated. The process has been extensively studied in the brain, the kidney, and the heart. Innate immunity, multiple signaling pathways involving cell survival and death, the unfolded protein response, autophagy, novel lipid mediators, mitochondria, and all signaling gases known somehow play a role (Fig. 1). We call the process ischemiareperfusion (IR) injury [1]. IR injury also features the preconditioning phenomenon. If the organ in question is deprived of blood supply only for a short time (usually less than $5 \mathrm{~min}$ ) several times in succession, the downstream cells are robustly protected from an extensive ischemic insult and IR injury is reduced. Preconditioning is nature's own homeopathy experiment (just a tiny bit of the same insult that causes the disease) to reduce IR injury. All information that we can gather regarding these processes is highly clinically relevant because although the research into IR injury and preconditioning has been extensive, the

F. C. Luft $(\bowtie)$

Experimental and Clinical Research Center,

Max-Delbrück Center and Charité Medical Faculty,

Lindenbergerweg 80,

13125 Berlin, Germany

e-mail: Luft@charite.de "translational" spinoffs relevant to clinical medicine have been modest.

The reperfusion injury salvage kinase pathway is the term given to a group of pro-survival protein kinases that include PI3K-Akt; MEK1/2 and Erk1/2 [2]. The mitogen-activated protein (MAP) kinase signaling pathway is an evolutionarily well-conserved route that transmits information from cell surface receptors to the cell nucleus (Fig. 2). MAP kinases also have important cytoplasmic functions in controlling translation and cell migration. Mammalian cells are outfitted with four groups, namely Erk1/2, Erk5, p38, and Jnk. Receptor tyrosine kinases commonly activate the Erk1/2 pathway through the action of guanine nucleotide exchange factors (Sos) that promote the loading of small G-proteins (Ras) with GTP. These GTPases then activate the threetiered kinase module consisting of MAP kinase kinase kinase (Raf), MAP kinase kinase (Mek1/2), and finally the MAP kinase (Erk1/2). The activation processes of Erk5, p38, and Jnk are less well described; however, the overall activation pathway appears to be similar to Erk1/2. The kinases seem to confer powerful cardioprotection, when activated specifically at the time of myocardial IR injury, thereby providing a possible pharmacological target for cardioprotection. However, other components including proteins involved in redox signaling, cardioprotective growth factors, and the mitochondrial permeability transition pore have also received attention [3].

In this issue, Beharier et al. [4] draw attention to a zinc transporter involved in IR injury. Their preliminary findings could bring to bear a fresh look at the entire IR injury spectrum of events and bring to bear a completely unexpected participant, namely ZnT-1. Palmiter and Findley cloned $\mathrm{Zn}-1$ in from a rat kidney cDNA expression library and proposed that ZnT-1 transports zinc out of cells to avoid zinc toxicity [5]. Since then, much more has been learned 
Fig. 1 Biological processes implicated in ischemia and reperfusion - the action and the planets. Adapted from [1]

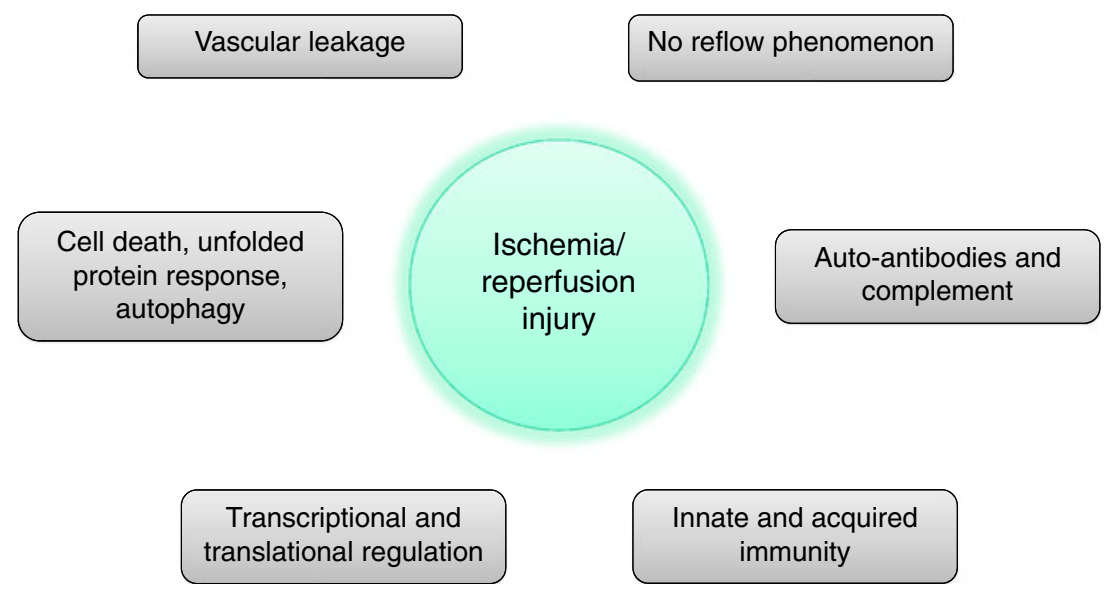

about this protein and the entire range of zinc transporters. The proteins all have transmembrane domains and are encoded by two solute-linked carrier gene families: $\mathrm{ZnT}$ (SLC30) and Zip (SLC39). There are at least $10 \mathrm{ZnT}$ and 15 Zip transporters in human cells. They appear to have opposite roles in cellular zinc homeostasis. $\mathrm{ZnT}$ transporters reduce intracellular zinc availability by promoting zinc efflux from cells or into intracellular vesicles, while Zip transporters increase intracellular zinc availability by promoting extracellular zinc uptake and vesicular zinc release into the cytoplasm. Both the ZnT and Zip transporter families exhibit unique tissue-specific expression, differential responsiveness to dietary zinc deficiency and excess, and differential responsiveness to physiologic stimuli via hormones and cytokines. Furthermore, the diseases influenced by zinc metabolism are vast $[6,7]$.

Beharier et al. were aware that ZnT-1 and its invertebrate homolog can activate Ras-Erk signaling. The mechanism involves binding of the ZnT-1 carboxy terminus to Raf-1 kinase, promoting its activation. Furthermore, the group showed an interaction between ZnT-1 and the L-type calcium channel. In earlier studies, they showed that rapid pacing, a non-specific stressor involving reactive oxygen species, upregulates ZnT-1. This constellation of findings led to their hypothesis that ZnT-1 could be involved in IR injury. The authors first relied on a cardiomyocyte cell model (HL-1 cells) from the AT-1 mouse atrial cardiomyocyte tumor lineage. HL1 cells can be serially passaged, yet they maintain the ability to contract and retain differentiated cardiac morphological, biochemical, and electrophysiological properties. Ultrastructural characteristics typical of embryonic atrial cardiac muscle cells were found consistently in the cultured HL-1 cells. Cyanide and aglycemia were used to simulate IR injury in the cells.

The authors found that ZnT-1 transfection and overexpression protected the cells from IR injury by measuring
Fig. 2 Schematic representation of MKP3-mediated regulation of Erk $1 / 2$ signaling. Commonly a receptor tyrosine kinase, through the action of guanine nucleotide exchange factors (Sos), small G protein Ras and MAP kinase kinase kinase (Raf), causes Mek-dependent degradation of mitogen-activated protein kinase phosphatase (MKP3), which leads to rapid increase in Erk1/2 phosphorylation. The mechanism is feed-forward. Concomitantly, Erk-dependent synthesis of MKP3 is induced, which at a later time point dephosphorylate Erk1/2. This mechanism provides negative feedback. The authors of this J Mol Med report [4] suggest that $\mathrm{ZnT}-1$ activates Raf in their model

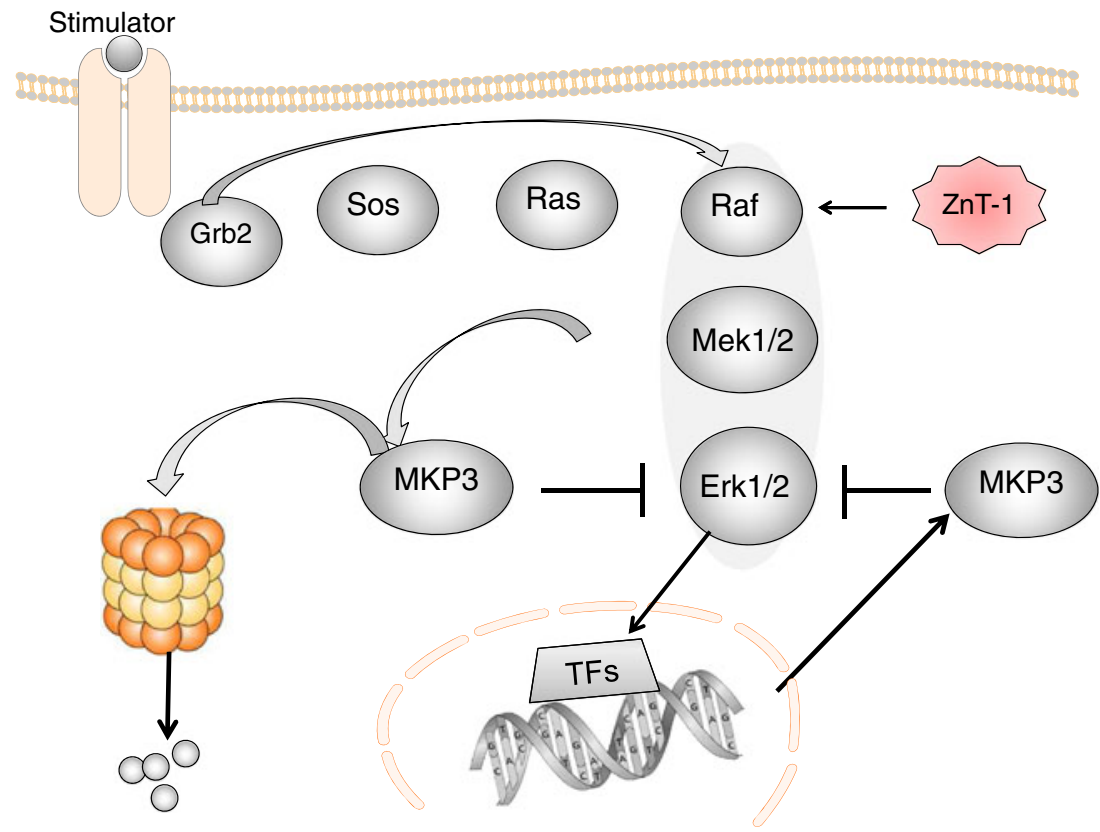


lactate dehydrogenase production and caspase activation. They then knocked down endogenous ZnT-1 and showed that IR injury was worsened. Beharier et al. also showed that ZnT-1 overexpression caused ERK1/2 phosphorylation, while the knockdown in nontransfected cells decreased ERK1/2 phosphorylation. A MEK inhibitor (PD98059) abrogated protection from IR injury by ZnT-1 overexpression. The investigators blocked L-type calcium channels to show that the known interaction between ZnT-1 and calcium channels was not responsible for the IR injury protection. Then, the authors produced a truncated ZnT-1 construct that lacked the $\mathrm{C}$ terminus. This construct could not interact with Raf1 and therefore would not activate ERK1/2. Subsequent$\mathrm{ly}$, the authors found that absence of the $\mathrm{C}$ terminus alone no longer provided protection from IR injury. However, when the authors expressed solely the $\mathrm{C}$ terminus (without the rest of the molecule), ERK1/2 was activated and the $\mathrm{C}$ terminus construct alone protected from IR injury when overexpressed. However, the $\mathrm{C}$ terminus construct could not pump zinc, and zinc exposure then killed the cells. Thus, the authors very cleverly separated zinc transport from IR injury protection. The authors next subjected rat hearts to IR injury. They observed a rapid upregulation of ZnT-1 with ischemia. During the reperfusion maneuver, ZnT-1 protein expression then decreased to a lower level.

The experiments are preliminary. You will surely be wondering where the invariable knockout mouse experiments are. However, ZnT1 is an essential gene, and homozygous knockout of the $\mathrm{ZnT} 1$ gene is lethal to the embryo [8]. Whether or not a conditional knockout strategy would be successful cannot be predicted for certain; an induction strategy could help in that regard. A transgenic strategy of overexpression could also be considered. Finally, the authors are not the first to tackle MAPK signaling as a strategy for IR injury protection. As they discuss, Schulman et al. used urocortin to activate MAPK signaling pharmacologically. Urocortin is a member of the sauvagine/corticotropin-releasing factor/urotensin I family of proteins. Schulman et al. showed that urocortin attenuated lethal reperfusion-induced injury both in vitro and in vivo via a p42/p44 MAPK-dependent mechanism [9]. In any event, IR injury and protection continue to fascinate; translation is desperately needed, and perhaps ZnT-1 will provide a key.

Respectfully,

Friedrich C. Luft

\section{References}

1. Eltzschig H, Eckle T (2011) Ischemia and reperfusion-from mechanism to translation. Nat Med 17:1391-1401

2. Hausenloy DJ, Yellon DM (2007) Reperfusion injury salvage kinase signaling: taking a RISK for cardioprotection. Heart Fail Rev 12:217-1234

3. Hausenloy DJ, Yellon DM (2009) Cardioprotective growth factors. Cardiovasc Res 83:179-194

4. Beharier O, Dror S, Levy S, Kahn J, Mor M, Etzion S, Gitler D, Katz A, Muslin AJ, Moran A, Etzion Y (2012) ZnT-1 protects HL-1 cells from stimulated ischemia-reperfusion through activation of Ras-ERK signaling. J Mol Med. doi:10.1007/s00109-011-0845-0

5. Palmiter RD, Findley SD (1995) Cloning and functional characterization of a mammalian zinc transporter that confers resistance to zinc. EMBO J 14:639-649

6. Liuzzi JP, Cousins RJ (2004) Mammalian zinc transporters. Annu Rev Nutr 24:151-172

7. Lichten LA, Cousins RJ (2009) Mammalian zinc transporters: nutritional and physiologic regulation. Annu Rev Nutr 29:153-176

8. Langmade SJ, Ravindra R, Daniels PJ, Andrews GK (2000) The transcription factor MTF-1 mediates metal regulation of the mouse ZnT1 gene. J Biol Chem 275:34803-34809

9. Schulman D, Latchman DS, Yellon DM (2002) Urocortin protects the heart from reperfusion injury via upregulation of $\mathrm{p} 42 / \mathrm{p} 44$ MAPK signaling pathway. Am J Physiol Heart Circ Physiol 283: H1481-H1488 